Fecha de recepción: septiembre 2019 Fecha de aceptación: noviembre 2019 Versión final: febrero 2020

\section{Proyecto TID: Transferencia - Innovación - Diseño para la Diversificación Productiva Exportadora de la Región del Biobío, Chile}

Resumen: Biobío es una de las regiones más importantes de Chile a nivel productivo. Fuente de recursos forestales, agropecuarios y pesqueros, polo de desarrollo industrial, de formación de capital humano e investigación, es una de las zonas con mayor proyección de crecimiento y descentralización. En este contexto, el Comité de Desarrollo Productivo Regional tiene como objetivo incrementar la capacidad innovadora y la competitividad del tejido empresarial, en especial de sus pequeñas y medianas empresas. Es aquí donde el diseño posee un espacio que no se ha formalizado como tal. La Facultad de Diseño de la Universidad del Desarrollo ha tenido como misión, desde sus comienzos, impulsar la realización de proyectos académicos que posibiliten a sus estudiantes estar en contacto directo con el emprendimiento y los procesos de desarrollo económico. Gracias a la adjudicación del Fondo de Innovación para la Competitividad Gobierno Regional del Biobío, al apoyo del Instituto de Innovación Interdisciplinaria iCubo UDD y ProChile Regional, a partir de 2014, nace el proyecto TID, el cual permitió fortalecer dicho vínculo y profundizar el trabajo colaborativo entre la universidad y el sector productivo. TID se planteó en su inicio como una experiencia piloto para fomentar la transferencia tecnológica entre academia y empresa local, a través de la disciplina del diseño. Su propósito fue contribuir a la visibilización de oportunidades para instalar el diseño como pieza clave en el engranaje de generación de innovación a nivel regional, aportando al cambio de malla curricular; a la formación de capital humano de diseñadores entrenados en innovación; a la implementación de infraestructura y tecnologías; a la ejecución de un programa piloto de transferencia de diseño a 12 empresas regionales; y a la implementación de diversas acciones de difusión del diseño a nivel regional y nacional como un catalizador de competitividad tanto en economías desarrolladas como emergentes. La participación de representantes regionales de instituciones académicas, gobierno, asociaciones gremiales y empresarios, y la transferencia de diseño a empresas, nos entregaron la oportunidad de contrastar visiones para un mejor entendimiento de la realidad de esta disciplina. Pero no solo eso, también nos dieron la posibilidad de obtener valiosos aprendizajes, que son la base para la propuesta de desafíos y responsabilidades a coordinar entre la triada Estado-Academia -Empresa (también denominada triple hélice), para incorporar al diseño en la Estrategia Regional de Innovación.

Dicha experiencia piloto fue el punto de partida para la formulación del proyecto TID UDD 2019-2021 "Programa TID Transferencia Innovación Diseño 2019-2021. Articulación Estado-Academia-Empresa para la diversificación productiva con base tecnológica y sustentable, de bienes y servicios exportables para empresas de la Región del Biobío" el 
cual ha sido financiado nuevamente por el Fondo de Innovación para la Competitividad de la Región del Bío Bío (FIC-R) 2018.

A través de este proyecto, se consolidará el programa TID y su inserción en la Estrategia Regional de Desarrollo del Biobío, buscando impactar en la investigación y desarrollo para la diversificación de bienes y servicios exportables, logrando un programa referente desde la región, hacia Chile y el resto del mundo.

El programa contempla poner en marcha tres áreas de trabajo:

1. Mesa TID: espacio de articulación, a través de encuentros mensuales de discusión presencial y toma de decisiones del Programa TID, con participación de distintos actores relevantes de la triple hélice a nivel regional.

2. Observatorio TID: espacio de investigación, diagnóstico y monitoreo de empresas de los distintos sectores productivos de la Región del Biobío, que se implementará en colaboración nacional con GEM Global Entrepreneurship Monitor Chile e internacional con el Institute for Design Innovation, London.

3. Laboratorio TID: espacio de investigación y desarrollo tecnológico que buscará incrementar la creación de valor de nuevos productos y servicios sustentables para empresas de base productiva de la Región del Biobío, a través de la articulación colaborativa con la triple hélice, con el apoyo del Área de Exploración Tecnológica UDD, y la Subdirección de Transferencia Tecnológica iCono UDD.

Además, se contempla la realización de eventos, exposiciones y seminarios abiertos a toda la comunidad empresarial, académica y de fomento productivo de la región.

Palabras clave: diseño - innovación - transferencia teconológica - desarrollo regional.

[Resúmenes en inglés y portugués en las páginas 260-262]

(1) Directora de Investigación de la Facultad de Diseño Universidad del Desarrollo. Diseñadora Industrial y magíster en Ingeniería Industrial de la Universidad del Bío-Bío. Máster en Dirección de Marketing y Gestión Comercial, Business \& Marketing School ESIC, España. Directora del Proyecto TID Transferencia-Innovación-Diseño, financiado por el Fondo de Innovación para la Competitividad del Gobierno Regional del Biobío.

(2) Diseñador de la Pontificia Universidad Católica de Chile. Coordinador de Extensión de la Facultad de Diseño de la Universidad del Desarrollo en Concepción. Coordinador del Proyecto TID Transferencia-Innovación-Diseño, financiado por el Fondo de Innovación para la Competitividad del Gobierno Regional del Biobío. 


\section{¿Por qué el TID?: Importancia del diseño como factor de desarrollo global, nacional y regional}

Hoy vivimos la llamada Revolución de la Información, que trae consigo el quiebre de muchos paradigmas y cambios acerca de lo que entendemos por necesidades básicas, calidad de vida, productos y servicios. Los importantes avances en telecomunicaciones, transportes, electricidad y tecnologías de la información, en general, junto al fenómeno de la Aldea Global (McLuhan, 1993), han obligado a las empresas a cambiar sus estructuras organizacionales para dar frente a las nuevas exigencias del mercado (Lev \& Hand, 2003). En este escenario, lo intangible del valor diferenciador cobra un peso protagónico para la competitividad y el capital intelectual preparado para innovar representa la base de estos nuevos activos. Pero, ¿el sector productivo considera al diseño como parte de su estrategia para afrontar las nuevas exigencias del mercado? ¿Nuestro país reconoce al diseñador como un actor en el capital humano preparado para innovar? Organizaciones gubernamentales de países desarrollados, conscientes de que la gestión del diseño aporta a la innovación y competitividad, han generado mediciones, herramientas y planes que le han brindado un lugar, tales como The Design Economy Report (The Design Council, 2018) o Design Delivers (Danish Design Centre, 2018) dentro de los más relevantes, así como también, en economías emergentes, se están desarrollando acciones para incorporar esta disciplina en el ámbito productivo, como por ejemplo el estudio "Diseño en la Argentina. Estudio del impacto económico" (Instituto Nacional de Tecnología Industrial, 2008), sin embargo existe un precario levantamiento de datos en torno al impacto real del diseño en la economía y productividad en Chile. Un acercamiento parcial al tema es posible desde la perspectiva de la innovación: nuestro país ocupó el lugar 42 en el ranking mundial y el primer puesto a nivel latinoamericano, según de The Global Innovation Index 2015: Effective Innovation Policies for Development (Cornell University, INSEAD, \& WIPO, 2015), el cual reveló que Chile se encontraba en la fase de desarrollo económico con foco en la eficiencia, donde el siguiente paso es la innovación, tendencia predominante en economías desarrolladas. La Agenda de Innovación y Competitividad Chile 2010-2020 (Consejo Nacional de Innovación para la Competitividad, 2010) afirmó que si bien en Chile se ha generado una creciente valoración de la innovación, existen importantes desafíos para innovar en los sectores exportadores basados en recursos naturales: "[...] dos terceras partes de las empresas reconocen que no han realizado ningún tipo de innovación (producto, proceso, marketing, diseño o gestión)". Por otro lado, en Surfeando hacia el futuro. Chile en el horizonte 2025 (Consejo Nacional de Innovación para la Competitividad, 2013) se abordaron temáticas como: la necesidad de educar para el diseño y el emprendimiento; la emergencia del diseñador y el emprendedor; y el rol central del diseño en la creación de innovación en el escenario de la nueva economía. En la misma línea, Mapeo de las industrias creativas en Chile. Caracterización y dimensionamiento (Consejo Nacional de la Cultura y las Artes, 2014) mencionó que el diseño "[...] se ha expandido adquiriendo múltiples expresiones creativas y penetrando como un elemento sustancial en la producción de diversos sectores económicos". A su vez, en Chile Creativo (Parada, Vergara, \& García Mintz, 2016) el diseño se declaró como subsector competitivo de las industrias creativas, priorizado para el programa estratégico nacional de especialización inteligente para la competitividad. El 
libro Nuevos Creativos Chilenos. Volumen 1, Diseño de Productos (Fuentes, 2015) expuso hitos relevantes para el diseño nacional, como la creación de la Coordinación Nacional de Diseño dentro del actual Ministerio de las Culturas, las Artes y el Patrimonio (ex CNCA), dando el primer espacio institucional al diseño, abriendo una línea propia en el concurso del Fondo Nacional para el Desarrollo Cultural y las Artes (Fondart), desarrollando la reciente implementada Política de Fomento del Diseño 2017-2022 y articulando a otras entidades del Estado como ProChile, la Dirección de Asuntos Culturales (Dirac) del Ministerio de Relaciones Exteriores y el Ministerio de Economía, Fomento y Turismo, los cuales han aportado recursos importantes a la hora de proyectar internacionalmente las marcas y estudios de diseño. Así también, la publicación de Diseño, Motor de transformación (Asociación de Empresas de Diseño, ChileDiseño, 2015) abrió conversaciones sobre el rol estratégico de la disciplina en cuatro sectores económicos del país: agroindustria, educación, retail y salud, reconociendo que: "Transitamos hacia una era de transformación, donde el éxito de los productos, servicios y organizaciones depende cada vez más del valor agregado por el diseño". En esta línea, la Asociación Chilena de Diseño está desarrollando acciones para reunir el diseño nacional en una red de colaboración, buscando posicionarlo como un factor clave en el crecimiento económico y social de Chile.

\section{El diseño desde las oportunidades de cada región}

La disparidad económica y social existente entre las regiones de Chile, requiere abordar el desafío de incorporar el diseño desde la comprensión de las prioridades estratégicas de su contexto local. La Región del Biobío es un referente de oportunidades para el diseño. Es un territorio altamente industrializado, participa del 9-10\% del PIB nacional, es responsable aproximadamente del $9 \%$ de las exportaciones chilenas y es el segundo centro industrial de Chile, después de Santiago. Posee una amplia base de sectores productivos, siendo una de las regiones del país más diversificadas en su estructura productiva (OCDE, 2009). El $60 \%$ de lo producido se exporta, siendo la región con mayor grado de apertura y vinculación con mercados internacionales, lo que conlleva a un tipo de desarrollo económico importante para Chile, pero centrado en la explotación de materias primas. En la Síntesis Regional del Consejo Nacional de la Cultura y las Artes (Consejo Nacional de la Cultura y las Artes, 2015) se expuso que la zona cuenta, además, con un desarrollo económico que supera la mera exportación de recursos naturales, pues se potencia con la industria y los servicios. Sin embargo, evidencia una baja capacidad de innovar por parte de las Pymes. La Estrategia Regional de Innovación 2012-2016 Región del Biobío. Diseño y establecimiento de la Estrategia Regional de Innovación y acciones afines en Biobío (Aliasgroup, 2014) señalaba que la zona posee una potencia tecnológica limitada e insuficiencia en su gestión de innovación, y declaraba una oportunidad en "[...] las nuevas formas de innovación, el estímulo a la creatividad, y la concepción de diseño (design thinking)". Dentro de este panorama general y, según lo enunciado en la cuenta pública regional de 2015, se presenta un importante potencial de desarrollo y crecimiento. Así mismo, la Estrategia Regional de Desarrollo de la Región del Biobío 2015-2030 (División de Planificación y Desa- 
rrollo Regional del Gobierno Regional del Biobío, 2015) de la Región del Biobío proyecta: "[...] una región innovadora y competitiva, con una mayor diversidad productiva que dé oportunidades de trabajo para todos", fomentando el desarrollo de investigación aplicada vinculada a las necesidades productivas de la zona. Es aquí donde el diseño presenta una oportunidad no explotada como herramienta estratégica, tanto para liderar procesos interdisciplinarios de innovación en la industria chilena como para aportar a la descomoditización, ampliando la oferta de productos, servicios y experiencias para acceder a los exigentes mercados internacionales. Pero, ¿qué opinan los diversos actores regionales respecto de la disciplina del diseño? ¿Cuáles son los principales desafíos a coordinar entre Estado, academia y empresa, para incorporar el diseño como disciplina en la Estrategia Regional de Innovación y Desarrollo en la Región del Biobío?

\section{Desafíos para incorporar el diseño en la estrategia regional de innovación del Biobío}

Con el objetivo de indagar y proponer un primer acercamiento a los desafíos para incorporar el diseño en la Región del Biobío, el equipo TID desarrolló un estudio de percepción en base a entrevistas y cuestionarios que convocó a una serie de representantes regionales de los ámbitos público y privado. En este, se incluyó a instituciones académicas, de Gobierno, asociaciones gremiales y empresarios, entre los cuales participaron Corfo, ProChile, HUB TEC, Industrias Creativas, Cidere Biobío, Corma, Socabio, Carnes JD, Versluys, Mar de Lagos, la Universidad del Bío-Bío, Duoc y la Universidad del Desarrollo. Este estudio, sumado a los resultados de la experiencia de transferencia de diseño a empresas, se convirtió en la base para la construcción de la propuesta de desafíos y responsabilidades a coordinar entre la triada Estado - academia - empresa para la incorporación del diseño en la Estrategia Regional de Innovación del Biobío, los cuales se exponen en detalle en la publicación Memoria TID 2014-2017. https://diseno.udd.cl/descarga-publicacionmemoria-tid-2014-2017/

Las conclusiones de este estudio preliminar plantean la necesidad de crear el Observatorio TID 2020, una instancia formalizada para liderar la medición y visibilización del aporte del diseño desde la academia en coordinación con Estado y Empresa. Considerando la conformación de un equipo de trabajo responsable del proyecto de investigación a largo plazo, dedicado al levantamiento, análisis, procesamiento de información y su difusión. La idoneidad del equipo ha sido fundamental, por esto se gestionó la colaboración nacional con GEM Global Entrepreneurship Monitor Chile e internacional con el Institute for Design Innovation, London, lo cual será fundamental en la búsqueda de objetivos plausibles en el tiempo, que permitan administrar los avances, posibles cambios y crecimientos del estudio, así como contar con la capacidad de levantar fondos de financiamiento, fortalecer las redes de cooperación, apoyar en la generación de insumos para la difusión y, sobre todo, capacidad para consolidar en resultados concretos la información obtenida de los procesos de investigación. Se proyecta la implementación del Observatorio TID para el mes de abril del año 2020 . 


\section{Transferencia de diseño a empresas de la región del Biobío}

Uno de los desafíos de TID fue generar una mirada pragmática de la realidad productiva de las pequeñas y medianas empresas (Pymes) a nivel regional, evaluando los potenciales de innovación instalados. La zona posee una amplia base de sectores productivos, pero una baja concentración de empresas del ámbito creativo. Asimismo, es evidente la desconexión que existe entre la necesidad de innovar de las Pymes y el diseño. La principal razón es que: este último solo es percibido en relación con lo estético, desconociendo su potencial estratégico y metodológico para liderar procesos interdisciplinarios de innovación. Existe un bajo nivel de colaboración entre estos sectores, lo que dificulta el acceso a capital humano para innovar y su especialización. Sin embargo, gracias a la competitividad, la innovación para la exportación de productos y servicios de Chile hacia el mundo es una perspectiva que va tomando fuerza en instituciones tanto públicas como privadas, presentándose como una buena oportunidad para generar vínculos de mutua colaboración. Biobío es fuente de recursos forestales, agropecuarios y pesqueros. Concentra el $28 \%$ de la superficie nacional dedicada a cultivos, con una diversidad de actividades silvoagropecuarias y condiciones agroecológicas favorables para el desarrollo de productos hortofrutícolas y orgánicos, propiciando una interesante actividad vitivinícola local. Su desarrollo ganadero aporta un $10 \%$ de la producción nacional de carne. Además, posee un sector pesquero acuícola industrial que registra un total de 120 empresas exportadoras de productos del mar. En la zona, la actividad agroindustrial exportadora es creciente, así como la infraestructura para su procesamiento. Frente a este escenario, se presentan grandes desafíos para la región, con planes sectoriales que apuntan a la diversificación de la oferta exportable, agregar valor a sus productos y desarrollar innovaciones que permitan competir con éxito en los mercados internacionales. Así también, el Comité de Desarrollo Productivo Regional del Biobío tiene entre sus objetivos incrementar la capacidad innovadora y la competitividad del tejido empresarial, en especial de sus pequeñas y medianas empresas. Es aquí donde el diseño presenta un potencial no explotado como herramienta estratégica para liderar procesos interdisciplinarios de innovación en las empresas, y donde se funda el programa de transferencia de diseño a empresas. Buscando reunir tanto a instituciones del Estado como a la empresa y la academia, la Facultad de Diseño de la Universidad del Desarrollo invitó a ProChile Regional como institución colaboradora y vinculante con el sector productivo exportador, convocando en conjunto a empresarios regionales dispuestos a la exploración de oportunidades para innovar desde el diseño. Las Pymes e instituciones elegidas representaron distintos tamaños de empresa, sectores productivos y nivel de desarrollo exportador: Orquídeas Bío Bío, las viñas Santa Berta y Vinos Pandolfi Price, comprendiendo productos hortofrutícolas orgánicos; Recetario Nativo, del sector agroindustrial; Kepika y Chile Merkén, de especies; Carnes JD, cárnicos; Mar de Lagos, del sector pesquero industrial; Roggendorf y Versluys, de la industria alimentaria del rubro panadería, repostería y chocolatería; y por último, Sono y las instituciones sociales con la agrupación solidaria Médicos del Aire representando al sector de servicios. A nivel académico, el proyecto planteó un nuevo nivel de exigencia y modelo metodológico de trabajo interdisciplinario, que integró el uso activo de nuevas tecnologías, asesorías de expertos por área, procesos de observación en terreno para el levantamiento de datos, jor- 
nadas de debate, análisis y evaluación. Todo ello requirió de un alto nivel de compromiso tanto de los docentes como de los alumnos de Diseño UDD y dLab, lo que fue fundamental para implementar las acciones de formación de capital humano y liderar pilotos de transferencia de diseño en empresas regionales. Es destacable la participación activa de los docentes Carolina Huilcamán, Ignacio García, Lorena Sanhueza, César Sagredo, Ángela Ferrada, Paola Silvestre, Rodrigo Caparrós, Cristian Lama y Macarena Vera. El programa de transferencia y capacitación de alumnos permitió: explorar acciones de difusión del valor del diseño al interior de las compañías; conectar la formación académica con desafíos y necesidades locales; y aplicar tecnologías de prototipado rápido, fabricación digital y métodos de innovación para el desarrollo de propuestas de diseño interdisciplinarias. Esta primera versión del TID fue planteada como una experiencia piloto, que ha significado un diagnóstico preliminar con resultados y aprendizajes que relevan aspectos cruciales a considerar, para la continuidad del programa y sus posibles lineamientos futuros. Las etapas de diagnóstico y transferencia del proyecto, confrontando la dualidad teoría-práctica, han otorgado la oportunidad única de contrastar visiones para un mejor entendimiento de la realidad del diseño en la Región del Biobío, y que fueron el punto de partida del planteamiento y futura implementación del Laboratorio TID 2021, como espacio de investigación y desarrollo tecnológico que buscará incrementar la creación de valor de nuevos productos y servicios sustentables para empresas de base productiva de la Región del Biobío, a través la articulación colaborativa (Estado-Academia-Empresa), con el apoyo de las unidades de Exploración Tecnológica Área IX UDD, y la Subdirección de Transferencia Tecnológica iCono UDD, además de la colaboración de instituciones extranjeras. A través del uso de tecnologías y metodologías de última generación, el Laboratorio TID trabajará en la innovación conjunta para potenciar la competitividad y la apertura a nuevos mercados a nivel nacional e internacional. Abriendo esta oportunidad a empresas de los diversos sectores productivos regionales, en una convocatoria abierta para la selección de 50 empresas, 10 mentores, 200 docentes y alumnos, que serán parte de un proceso de innovación conjunta, concursando por la implementación referente de 5 proyectos para la exportación. Contando además con seminarios abiertos a toda la comunidad empresarial, académica y de fomento productivo de la Región.

\section{Tiempos de diseño}

En mayo de 2017, se lanzó el Plan Nacional de Economía Creativa, que tiene por objetivo hacer de este ámbito, dentro del cual se encuentra el diseño, un motor de desarrollo social, cultural y económico para Chile. Su misión es lograr la articulación entre las distintas instituciones del Estado y con los privados para generar subsidios, apoyo en materia de comercialización y simplificación de trámites, y contribuir a la diversificación productiva, agregando valor a las exportaciones. En coherencia con ello, el 18 de diciembre de 2017, inició la implementación de la Política Nacional de Fomento del Diseño 2017-2022, que reconoce a esta disciplina como un sector que cumple un papel estratégico en la generación de valor y diferenciación de las organizaciones, en el crecimiento económico de un 
país, y en la consolidación y difusión de su imagen y cultura. Es claro que el diseño es una disciplina en pleno desarrollo. En junio de 2017, la Asociación Chilena de Empresas de Diseño, Chile Diseño, junto a GFK-Adimark realizó una auditoría que le permitió develar un crecimiento de $20 \%$ en 2016 , de las 40 principales agencias, estudios y empresas que configuran la industria del diseño en el país. Pese a este prometedor panorama, aún existe un obstáculo difícil de vencer y que es, junto a la falta de financiamiento, la principal barrera que experimentan las pequeñas y medianas empresas a la hora de pensar en el diseño como aliado natural en la innovación: el desconocimiento. Bajo esa necesidad, la Facultad de Diseño de la Universidad del Desarrollo desde sus inicios ha impulsado el trabajo académico de sus docentes y alumnos con instituciones públicas y privadas, y dentro de este contexto, el programa de transferencia realizado entre 2014 y 2017 con 12 empresas regionales ha sido uno más de los esfuerzos que ha permitido el registro y difusión de estas experiencias. Gracias al financiamiento del Gobierno Regional, el proyecto TID significó un acercamiento en la integración entre el diseño y el sector productivo de la Región del Biobío. Se constituyó como una experiencia piloto de aprendizajes y planteamiento de desafíos para la construcción de un ecosistema de innovación regional donde el diseño sea parte fundamental. Dentro de estos aprendizajes, se estableció que el apoyo del Estado es esencial para generar espacios de colaboración y diálogo entre la academia y las empresas, la creación de programas específicos que integren diseño y sector productivo en un trabajo sistemático y descentralizado, que aporte al desarrollo de ecosistemas productivos regionales autosustentables, autónomos y con valor territorial. Asimismo, se determinó que debe existir un trabajo conjunto entre los organismos estatales y la academia para la construcción de un plan de difusión, cuyo fin es relevar el valor del diseño como herramienta estratégica para el desarrollo del Biobío. En tanto, la academia debe actualizar sus mallas curriculares y capacitar a sus docentes, alineada con las nuevas competencias y tecnologías, para generar capital humano con capacidad de trabajo interdisciplinario, empoderado con los desafíos regionales y globales y con foco en la innovación. Es necesario comprometer una labor cohesionada a la del sector productivo, que permita que este abra sus puertas para integrar el diseño en su modelo de negocios, y aportar a la construcción de métricas sobre la rentabilidad de su empresa. Estos son solo algunos de los desafíos que se pudieron visualizar gracias al proyecto TID, cuya resolución dependerá principalmente de cómo colaboren entre sí los actores de la triada Estado-academia-empresa y, por otro lado, para la Facultad, se espera que se sigan desarrollando proyectos a través del programa RED (Relación Empresa Diseño), recientemente lanzado por Diseño UDD, para fortalecer la estructura metodológica de la ejecución de los proyectos colaborativos, que permiten la transferencia desde la universidad a distintos sectores productivos y sociales. De manera complementaria, y como parte de la segunda etapa del programa TID UDD 2019-2021, se contempla la construcción y formalización del Observatorio TID 2020 de impacto del Diseño en el sector productivo regional el cual se plantea como proyecto de investigación a largo plazo, orientado al levantamiento y sistematización de casos de éxito y experiencias referentes del diseño como aporte a la economía local, buscando revelar sus resultados y potenciales con una mirada cuantitativa. Su objetivo principal es contribuir a la generación y difusión de conocimiento del diseño como herramienta estratégica aplicada, el cual permita validar su real aporte y así determinar las acciones estratégicas más per- 
tinentes para implementar de manera práctica a través del Laboratorio TID 2021, como espacio de desarrollo tecnológico que buscará incrementar la creación de valor de nuevos productos y servicios sustentables para empresas de la Región del Biobío, impactando en el ecosistema productivo regional a mediano y largo plazo.

\section{Bibliografía}

McLuhan, Marshall (1993). La aldea global. Barcelona: Gedisa.

Lev, B. \& Hand, J. R. (2003). Intangible Assets : Values, Measures, and Risks. Nueva york: Oxford University Press.

The Design Council (2018). The Design Economy 2018 report. Recuperado de https://www. designcouncil.org.uk/resources/report/design-economy-2018

Danish Design Centre (2018). Design Delivers 2018, How design accelerates your business. Recuperado de https://danskdesigncenter.dk/en/design-delivers-2018-how-designaccelerates-your-business

Instituto Nacional de Tecnología Industrial (2009). Diseño en la Argentina, estudio del impacto económico 2008. Instituto Nacional de Tecnología Industrial, Programa de Diseño - Buenos Aires: INTI.

Cornell University, INSEAD, and WIPO. (2015). The Global Innovation Index 2015: Effective Innovation Policies for Development. Switzerland: World Intellectual Property Organization.

Consejo Nacional de Innovación para la Competitividad. (2010). Agenda de Innovación y Competitividad Chile 2010-2020. Chile: Consejo Nacional de Innovación para la Competitividad.

Consejo Nacional de Innovación para la Competitividad. (2013). Surfeando hacia el futuro. Chile en el horizonte 2025. Chile: Consejo Nacional de Innovación para la Competitividad.

Consejo Nacional de la Cultura y las Artes (2014). Mapeo de las industrias creativas en Chile. Caracterización y dimensionamiento. Chile: Consejo Nacional de la Cultura y las Artes.

Parada, J.; Vergara, M. L. \& García Mintz, Á. (2016). Chile Creativo. Chile: Estratégica.

Fuentes, J. P. (2015). Nuevos Creativos Chilenos. Volumen 1, Diseño de Productos. Chile: Consejo Nacional de la Cultura y las Artes.

Asociación de Empresas de Diseño, ChileDiseño (2015). Diseño, Motor de transformación. Chile: ChileDiseño.

OECD/Bío Bío's Regional Steering Committee. (2009). The Bío Bío Region, Chile: SelfEvaluation Report. OECD Reviews of Higher Education in Regional and City Development. Recuperado de www.oecd.org/edu/imhe/regionaldevelopment

Consejo Nacional de la Cultura y las Artes (2015). Región del Biobío. Síntesis Regional. Chile: Consejo Nacional de la Cultura y las Artes.

Aliasgroup. (2014). Estrategia Regional de Innovación 2012-2016 Región del Biobío. Diseño y establecimiento de la Estrategia Regional de Innovación y acciones afines en Biobío. Chile: Aliasgroup. 
División de Planificación y Desarrollo Regional del Gobierno Regional del Biobío (2015). Estrategia Regional de Desarrollo de la Región del Biobío 2015-2030. Chile: Gobierno Regional del Biobío.

Consejo Nacional de la Cultura y las Artes (2017). Plan nacional de fomento a la economía creativa. Chile: Consejo Nacional de la Cultura y las Artes.

Consejo Nacional de la Cultura y las Artes (2017). Política de Fomento del Diseño 2017-2022.

Chile: Consejo Nacional de la Cultura y las Artes.

\begin{abstract}
Biobío is one of the most important productive regions of Chile. Source of forest, agricultural and fishery resources, industrial development center, human capital formation and research, and one of the areas with the highest projected growth and decentralization.

In this context, the Committee on Regional Productive Development aims to increase the innovative capacity and competitiveness of the business fabric, especially of small and medium-sized businesses.

It is here where design has a space that has not been formalized as such. The Design School of Universidad del Desarrollo has had as its mission, since its inception, to impulse the conduction of academic projects that enable its students to be in direct contact with entrepreneurs and the processes of economic development.

Thanks to the grant of the Innovation Fund for Biobío Regional Government Competitiveness, the support of the Institute of interdisciplinary Innovation iCubo of UDD and ProChile, the TID Project started in 2014, which led to strengthen the link and deepen the collaborative work between the university and the productive sector. TID was initially created as a pilot experience to promote technology transfer between academia and local business, through the design discipline.

Its purpose was to contribute to the visibility of opportunities to install design as a key piece in the innovation generation gear at the regional level, contributing to the change of the curriculum; to the formation of human capital of designers trained in innovation; to the implementation of infrastructure and technologies; to the execution of a pilot design transfer program for 12 regional companies; and to the implementation of various actions to disseminate design at a regional and national level as a catalyst for competitiveness in both developed and emerging economies.

The participation of regional representatives of academic institutions, government, trade associations and businessmen, and the transfer of design to companies, gave us the opportunity to contrast visions for a better understanding of the reality of this discipline.

But not only that, they also gave us the possibility of obtaining valuable learning, which is the basis for the proposal of challenges and responsibilities to be coordinated between the triad State-Academy-Company (also called triple helix), to incorporate design into the Regional Strategy of Innovation.

This pilot experience was the starting point for the formulation of the TID UDD 20192021 project "TID Transfer Innovation Design 2019-2021 Program. Articulation State-
\end{abstract}


Academy-Company for the productive diversification with technological and sustainable base, of exportable goods and services for companies of the Biobío Region" which has been financed again by the Innovation Fund for Competitiveness of the BíoBío Region (FIC-R) 2018.

Through this project, the TID Program and its insertion in the Biobío Regional Development Strategy will be consolidated, seeking to impact research and development for the diversification of exportable goods and services, becoming a reference program from the region, to Chile and the rest of the world.

The program contemplates launching three work areas:

1. TID Table: articulation space, through monthly meetings of face-to-face discussion and decision making of the TID Program, with the participation of different relevant triple helix actors at the regional level.

2. TID Observatory: research, diagnosis and monitoring space for companies from the different productive sectors of the Biobío region, which will be implemented in national collaboration with GEM Global Entrepreneurship Monitor Chile and internationally with the Institute for Design Innovation, London.

3. TID Laboratory: research and technological development space that will seek to increase the creation of value of new sustainable products and services for productive-based companies in the Biobío Region, through collaborative articulation with the triple helix, with the support of the UDD Technology Exploration Area, and the Sub directorate of Technology Transfer iCono UDD.

In addition, the project contemplates organizing events, exhibitions and seminars open to the entire business, academic and productive development community of the region.

Keywords: design - innovation - technological transfer - Regional development.

Resumo: Biobío é uma das regiões mais importantes do Chile em nível produtivo. Fonte de recursos florestais, agrícolas e pesqueiros, pólo de desenvolvimento industrial, formação e pesquisa de capital humano, é uma das áreas com maior projeção de crescimento e descentralização. Nesse contexto, o Comitê Regional de Desenvolvimento Produtivo visa aumentar a capacidade inovadora e a competitividade do tecido empresarial, especialmente de pequenas e médias empresas. É aqui que o design possui um espaço que não foi formalizado como tal. A Faculdade de Design da Universidade de Desenvolvimento tem como missão, desde a sua criação, promover a realização de projetos acadêmicos que permitam que seus alunos tenham contato direto com os processos de empreendedorismo e desenvolvimento econômico. Graças ao prêmio do Governo Regional do Fundo de Inovação para a Competitividade da Biobío, ao apoio do Instituto de Inovação Interdisciplinar iCubo UDD e ProChile Regional, a partir de 2014, nasceu o projeto TID, que permitiu fortalecer esse vínculo e aprofundar o trabalho colaborativo, entre a universidade e o setor produtivo. O TID foi inicialmente criado como uma experiência piloto para promover a transferência de tecnologia entre a academia e as empresas locais, por meio da disciplina de design. Seu objetivo era contribuir para a visibilidade de oportunidades para instalar o design como peça chave nos equipamentos de geração de inovação em nível regional, contribuindo para a mudança da malha curricular; à formação de capital humano de 
designers treinados em inovação; à implementação de infraestrutura e tecnologias; à execução de um programa piloto de transferência de projeto para 12 empresas regionais; e à implementação de várias ações de disseminação do desenho nos níveis regional e nacional como catalisador da competitividade nas economias desenvolvidas e emergentes. A participação de representantes regionais de instituições acadêmicas, governo, associações comerciais e empresários e a transferência de design para empresas nos deu a oportunidade de contrastar visões para uma melhor compreensão da realidade dessa disciplina. Mas não apenas isso, eles também nos deram a possibilidade de obter um aprendizado valioso, que é a base para a proposta de desafios e responsabilidades de coordenação entre a tríade Estado-Academia-Empresa (também chamada de tripla hélice), para incorporar o design à Estratégia. Regional de Inovação.

Palavras chave: design - inovação - transferência de tecnologia - desenvolvimento regional.

[Las traducciones de los abstracts fueron supervisadas por el autor de cada artículo] 